\title{
Numerical simulation of coherent turbulent structures and of passive scalar dispersion in a canopy sub-layer
}

\author{
K. Gavrilov ${ }^{\text {a }}$, D. Morvan ${ }^{\text {b, } *}$, G. Accary ${ }^{\text {c }}$, D. Lyubimov ${ }^{\mathrm{a}}$, S. Meradji ${ }^{\mathrm{d}}$ \\ a Theoretical Physics Department, Perm State University, Perm, Russia \\ ${ }^{\mathrm{b}}$ M2P2, CNRS-Université d'Aix-Marseille, Marseille, France \\ ${ }^{\mathrm{c}}$ Holy-Spirit University of Kaslik, Jounieh, Lebanon \\ ${ }^{\mathrm{d}}$ IMATH, Université du Sud Toulon-Var, France
}

\begin{abstract}
This study deals with the problem of turbulent atmospheric boundary-layer flow over a forest canopy. Numerous previous works showed that this flow presents more similarities with a mixing-layer flow than with the standard boundary-layer flow. In this paper, this problem was studied for homogeneous canopies, using large eddy simulation (LES). The numerical results reproduced correctly the various steps of development of this flow: the appearance of a first generation of coherent structures resulting from the development of a primary Kelvin-Helmholtz instability, the reorganization of these structures, by vortex pairing and kinking, the development of a secondary instability and the formation of horseshoe vortices. Then, the process of transport of a passive scalar from a forest canopy into a clear atmosphere was studied in two cases, i.e., when the passive scalar concentration at the surface foliage is either constant or time-varying. Even though this small difference has little influence on the concentration patterns, the results showed that it can significantly affect the concentration magnitude as well as the dynamics of the total concentration in the atmosphere.
\end{abstract}

\section{Introduction}

The interest concerning the problem of atmospheric boundarylayer/canopy interaction has increased these last decades, in order to improve the knowledge of many environmental problems, such as the evaluation of heat and mass transfer between various vegetation covers and atmospheric surface layers, the impact of wind (especially gusts) upon tree plantations, or the effects of wind on wildfires behavior. The presence of canopy affects the height of the roughness sub-layer (RSL), which is nearly equal to three times the height of trees. The turbulence properties in the upper inertial sub-layer (ISL) of the atmospheric boundary layer (ABL), exhibit a tendency to a universal behavior that could be described by the Monin-Obuhov similarity theory [1,2]. Since there is no exhaustive model for turbulent flows in the RSL, the investigation of this kind of flow has a considerable scientific interest from a theoretical point of view.

Various experimental works, summarized in [3], have shown that statistics properties of a turbulent atmospheric boundarylayer flow over a forest canopy have common features with a turbulent mixing-layer flow. The drag forces induced by the constituting elements of the canopy (foliage, branches, twigs, etc.) slow

\footnotetext{
* Corresponding author.

E-mail address: dominique.morvan@univ-amu.fr (D. Morvan).
}

down the wind flow within the vegetation. The resulting action of these forces induces a significant shear of the free wind flow above the canopy in a similar manner as the situation observed in a mixing-layer flow. The transition between boundary layer and mixing-layer type flow can be characterized using a non-dimensional parameter introduced by Massman in [4]; this parameter was defined as the product between the average dragcoefficient $C_{D}$ (characterizing the action of the canopy upon the flow) and the leaf-area index (LAI). As it was reported by Ghisalberti and Nepf in [5] for aquatic canopies, the mixing layer regime is observed when the following threshold criterion is verified: $C_{D}$ $\times$ LAI $>0.1$. In this case, it was observed that the decay of turbulence inside the canopy exceeded the standard value of $-5 / 3$ predicted by the Kolmogorov theory. The coherent structures, formed above a forest cover, penetrate within the canopy and interact rapidly with the irregular grid formed by the vegetation. Consequently a part of the turbulent kinetic energy is directly injected at smaller scales, in short-cutting the inertial cascade and promoting the production of a wake kinetic energy [2]. The shear layer resulting from the action of drag forces, induced by the presence of vegetation, promotes the development of a KelvinHelmholtz $(\mathrm{KH})$ instability, characterized by the formation of large scale 3D coherent structures [2]. The evolution in space and in time of these vortices (vortex kinking and pairing) causes the transition to a mixing-layer type flow. Nevertheless, at the same time, typical 
boundary layer coherent structures, like horseshoe vortices, appear also in this flow.

One of the first investigations of coherent structures in canopy turbulence was proposed by Denmead and Bradley in 1987 [6]; they showed that time evolutions of different scalars such as temperature, humidity, and passive scalar concentration indicate the presence of specific 'ramp structures'. These structures were characterized by intensive gusts (sweeps) that penetrate within the canopy. When such a ramp structure crossed a given location, one could observe a sharp jump in the scalar signal, as it was shown in [6]. The spatial patterns in coherent turbulence structures was also studied in 1989 by Gao et al. [7] who used turbulence time-traces measured at different levels within and above forests, to reconstruct these spatial patterns.

Compared to Reynolds-Average Navier-Stokes (RANS) approach, large eddy simulation (LES) can be considered as a preferable technique of turbulence modeling, to capture the behavior of coherent large-scale structures in ABL flow. LES for ABL flows was first introduced by Deardorff in 1972 [8], followed some years later by Moeng in 1984 [9]. The first attempt to conduct LES for atmospheric flows over a forest canopy was done by Shaw and Schumann in 1992 [10]. In their work, the forest canopy was represented as a porous continuum medium, affecting the flow by means of a volume-distributed drag force. Using this approach, the coherent structures of a turbulent flow over a crop in the early stages of development, were simulated and discussed by Kanda and Hino in 1994 [11]. Ramp signals in scalar time-traces were highlighted, as the signature of passages of coherent turbulence structures [12]. Analysis of experimental data based on proper orthogonal decomposition (POD), allowed to reconstruct the velocity field of a 'characteristic eddy' (large coherent structure). This one consisted of a pair of counter-rotating streamwise vortices centered above the canopy. A strong gust or sweep motion generated between the vortices was responsible for most of the shear stress carried by large eddies. Recently, LES of passive scalar transported by advection though ABL above a canopy, strongly connected to ramp structures and to large scale vortices, were performed by Fitzmaurice et al. in 2004 [13] and Watanabe in 2004 [14]. The simulations reproduced in a quite realistic manner, the main characteristics of canopy turbulence, including typical ramp structures appearing in the time traces of a scalar concentration near the canopy top. Very recently, extractions of coherent structures from LES results, with the help of a POD analysis, were carried out by Huang et al. in 2009 [15]. And finally, a major contribution concerning the mechanisms of formation of coherent structures, based on LES results, was proposed by Finnigan et al. in 2009 [16]. As it was shown in these papers, the characteristic eddies consisted of an upstream head-down sweep-generating hairpin and of a vortex, superimposed to a downstream head-up ejection-generating hairpin. The conjunction of sweeps and ejections induced pressure maxima between hairpins, where were also located coherent scalar micro-fronts.

The present study deals with numerical simulations (based on a LES approach) of the interaction between a neutral atmospheric boundary layer (ABL) with a homogeneous canopy, and the exchange and transport of a passive scalar between canopy and $A B L$. The numerical results allowed in reproducing the main features characterizing this kind of geophysical flow in a homogeneous configuration, corresponding to the configuration previously reported by Su et al. [17]. The coherent structures observed during the flow organization and various statistical moments were investigated. Furthermore, we have also explored the problem of transport of a passive scalar over a canopy starting from a clear atmosphere. The source term of passive scalar was assumed to be located at the surface of vegetation (foliage). The release/capture rate of passive scalar was assumed to be proportional to the difference between the bulk scalar concentration in the flow and a reference concentration held by the foliage. While in Watanabe [14], this reference concentration was supposed to be constant (thus constituting an infinite source of concentration for the flow), we also considered here the case where the scalar concentration contained by the canopy evolved in time according to a kinetic equation; thus modeling a more realistic pollutant transfer from a contaminated canopy into a clear atmosphere.

\section{Physical and mathematical model}

In taken into account of additional terms resulting from drag forces induced by the vegetation, and after filtering the fluctuations due to subgrid scale (SGS) structures, the equations governing the resolved-scale fluid flow can be written as follows [14] (where the angle bracket indicates the filtered value):

$\frac{\partial\left\langle u_{j}\right\rangle}{\partial x_{i}}=0$

$$
\begin{aligned}
\frac{\partial}{\partial t}\left(\rho\left\langle u_{i}\right\rangle\right)+\frac{\partial}{\partial x_{j}}\left(\rho\left\langle u_{i}\right\rangle\left\langle u_{j}\right\rangle\right)= & -\frac{\partial\langle P\rangle}{\partial x_{i}}+\mu_{L} \frac{\partial}{\partial x_{j}}\left(\frac{\partial\left\langle u_{i}\right\rangle}{\partial x_{j}}+\frac{\partial\left\langle u_{j}\right\rangle}{\partial x_{i}}\right) \\
& +\frac{\partial \tau_{i j}}{\partial x_{i}}-a_{L} C_{D} \rho|\langle u\rangle|\left\langle u_{i}\right\rangle
\end{aligned}
$$

The presence of solid elements composing the vegetation was represented in the momentum equations and the SGS kinetic energy by additional drag terms (proportional to the module of the average velocity) and characterized by two parameters: the leaf area density (LAD) $a_{L}$ and the average drag-coefficient $C_{D}$, characterizing the whole canopy, (assumed to be constant and equal to 0.15 as considered in [17] from the analysis of micrometeorological data in a deciduous forest). The momentum flux, resulting from the SGS structures (SGS model), was approximated by introducing the SGS kinetic energy $k$ and the average stress tensor. Initially proposed by Schumann [18], this model was then commonly used by the ABL community $[8,15]$ :

$$
\begin{aligned}
\tau_{i j} & =-\frac{2}{3} \rho k \delta_{i j}+\mu_{T}\left(\frac{\partial\left\langle u_{i}\right\rangle}{\partial x_{j}}+\frac{\partial\left\langle u_{j}\right\rangle}{\partial x_{i}}\right), \quad \mu_{T}=\rho C_{\mu} L_{\mathrm{SGS}} \sqrt{k}, \quad L_{\mathrm{SGS}} \\
& =(\Delta x \Delta y \Delta z)^{1 / 3}
\end{aligned}
$$

where $\mu_{T}$ represents the SGS eddy viscosity and $C_{\mu}=0.07$ as in [14]. The value of $L_{\mathrm{SGS}}$ characterizes the typical size of the SGS filtering length. In agreement with previous numerical model proposed for the same kind of problems [14], the SGS kinetics energy was calculated by solving the following transport equation:

$$
\begin{aligned}
\frac{\partial}{\partial t}(\rho k)+\frac{\partial}{\partial x_{j}}\left(\rho\left\langle u_{j}\right\rangle k\right)= & \frac{\partial}{\partial x_{j}}\left(\mu_{T}\left(\frac{\partial k}{\partial x_{j}}\right)\right)+\tau_{i j} \frac{\partial\left\langle u_{i}\right\rangle}{\partial x_{j}}-\rho C_{\varepsilon} \frac{k^{3 / 2}}{L_{S G S}} \\
& -2 a_{L} C_{D} \rho|\langle u\rangle| k
\end{aligned}
$$

The dissipation term (proportional to $k^{3 / 2}$ ) highlights two parameters: $C_{\varepsilon}$ (a constant equal to 0.93 ) and the SGS length $L_{S G S}$. The last term on the right hand side of this equation is a destruction term for the SGS kinetics energy budget, resulting from drag-force effects inside the canopy.

As far as the passive scalar is concerned, its concentration $C$ in the flow, is governed, as in [14], by the turbulent transport Eq. (5), in which the last term represents the mass flux between the atmosphere and the leaf surface, it is proportional to the wind speed, to the LAD, and to the difference between the bulk concentration in the flow and the value at the surface of the canopy.

$$
\frac{\partial}{\partial t}(\rho\langle C\rangle)+\frac{\partial}{\partial x_{j}}\left(\rho\left\langle u_{j}\right\rangle\langle C\rangle\right)=\frac{\partial}{\partial x_{j}}\left(\frac{\mu_{L}}{S c} \frac{\partial\langle C\rangle}{\partial x_{j}}\right)-c_{C} a_{L} \rho|\langle u\rangle|\left(\langle C\rangle-\left\langle C_{\mathrm{ref}}\right\rangle\right)
$$


Unlike the study conducted in [13], in the present study, the reference concentration in the canopy $C_{\text {ref }}$ may vary with time and is determined in that case from the following kinetic equation:

$\frac{\partial}{\partial t}\left(\rho\left\langle C_{\text {ref }}\right\rangle\right)=c_{C} a_{L} \rho|\langle u\rangle|\left(\langle C\rangle-\left\langle C_{\text {ref }}\right\rangle\right)$

On one side, if the passive scalar resulted from a metabolism mechanism, plant respiration for example, the concentration $C_{\text {ref }}$ can be considered as equal to a constant value. On the other side, if $C_{\text {ref }}$ represented the concentration of dusts or chemical compounds deposited at the surface of the foliage, its value resulted from a mass balance equation between the atmosphere and the surface of the foliage (Eq. (6)). To be consistent with Eq. (5), the exchange term on the right hand side of Eq. (6) was equal (with an opposite sign) to the same term appearing in Eq. (5).

In this study, the SGS Schmidt number $(S c)$ was equal to $1 / 3$ and the scalar exchange coefficient for the leaves $\left(c_{C}\right)$ was equal to 0.04 as in [14].

\section{Numerical method}

The transport equations governing the evolution of this geophysical system were solved using a fully-implicit finite volume method, and using a segregated algorithm, using a third order Euler scheme for the time integration and a PISO algorithm [19] for the pressure/velocity coupling. The numerical method was second order accurate in space: the QUICK scheme (third order) (with a flux limiting strategy) was used for the convective terms while a second-order central-difference scheme was used for the diffusive ones. The non-symmetric linear systems obtained from the discretized transport equations were solved using a BiCGStab iterative method, while the symmetric linear system of the pressure equation was solved using a Conjugate Gradient (CG) method preconditioned using the artificial compressibility technique. The developed code has been validated on several benchmark tests of natural and forced convection [20]. At each time-step, a converged solution was supposed to be obtained when the residuals of all transport equations reached the $L_{2}$-norm tolerance of $10^{-6}$ in non-dimensional form. The calculations were performed on a shared memory machine (SGI ALTIX), with 20 Itanium 2 processors and 40 GBytes memory, using OpenMP instructions for the loops parallelization $[20,21]$.

\section{Numerical results for the flow dynamic}

We consider first ABL flow over a homogeneous forest cover. The canopy height $H$ was equal to $20 \mathrm{~m}$ and a streamwise velocity equal to $12 \mathrm{~m} / \mathrm{s}$ was imposed at the upper boundary of the computational domain shown in Fig. 1a. Periodic boundary conditions were imposed in both $X$ and $Y$ directions and standard wall functions [22] were used at the bottom boundary for a better estimation of the shear-rate near the wall. The simulations were carried out for the following dimensions: $L_{X}=300 \mathrm{~m}, L_{Y}=100 \mathrm{~m}$, and $L_{Z}=80 \mathrm{~m}$. The number of grid points used for these calculations was equal to $150 \times 50 \times 80=600,000$, consequently the grid sizes along the three directions were $\Delta x=\Delta y=2 \mathrm{~m}$ and $\Delta z=1 \mathrm{~m}$. Along the vertical direction, assuming that above the canopy the integral length scale $L_{T}$ was nearly equal to the height of the canopy $H$ [2], the ratio $\Delta z / L_{T}$ was equal to 0.05 , which was enough to assume that the wave length of the eddy viscosity filter was located inside the inertial zone. The vertical distribution of the LAD is shown in Fig. $1 \mathrm{~b}$; the value of the leaf area index $(\mathrm{LAI}=2)$ indicates that the canopy was sparse enough in the considered case. The simulations conditions were chosen in order to fit the experimental conditions observed in a partially defoliated canopy in a deciduous
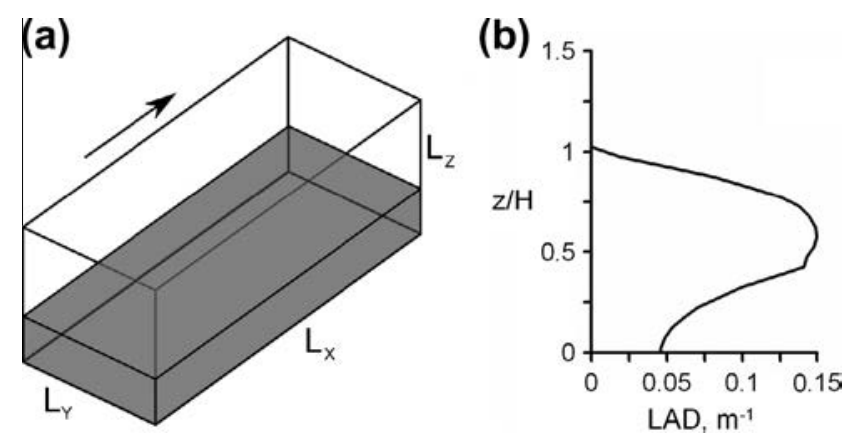

Fig. 1. Configuration under consideration: (a) the computational domain and (b) the leaf area density vertical distribution.

forest [23]. Note that for the results presented in this section, the passive scalar concentration was not computed, i.e. Eqs. (5) and (6) were not solved in this part.

The streamwise velocity profile was initialized using the same procedure as suggested in [24], in adding a white noise to accelerate the development of $\mathrm{KH}$ instability. In considering the time necessary to obtain the first coherent structures in the flow field, we can be sure that the numerical results were not affected by this initialization procedure.

The normalized vertical profile of the mean streamwise velocity $U(z) / U(H)$, shown in Fig. 2a, contains an inflection point around $z=H$. Hence, the shear is maximum around $z=H$, and its strength can be described by the shear length scale $L_{S}$ (Eq. (8)). This profile changes in time with the flow evolution and becomes straighter. Since for the present LES, $C_{D} \times \mathrm{LAI}=0.3$ is not large enough, the vertical profile of the mean streamwise velocity can be regarded as the superposition of a logarithmic boundary-layer profile and a mixing-layer one. Nevertheless, other dynamic characteristics such as the root-mean-square (RMS) velocities, $\sigma_{U}$ (Fig. 2b) and $\sigma_{W}$ (Fig. 2c), the Reynolds shear stress $\tau_{X Z}=-\left\langle u^{\prime} w^{\prime}\right\rangle$ (Fig. 2d), the skewness $S k_{U}$ (Fig. 2e), and $S k_{W}$ (Fig. 2f) show more analogy with
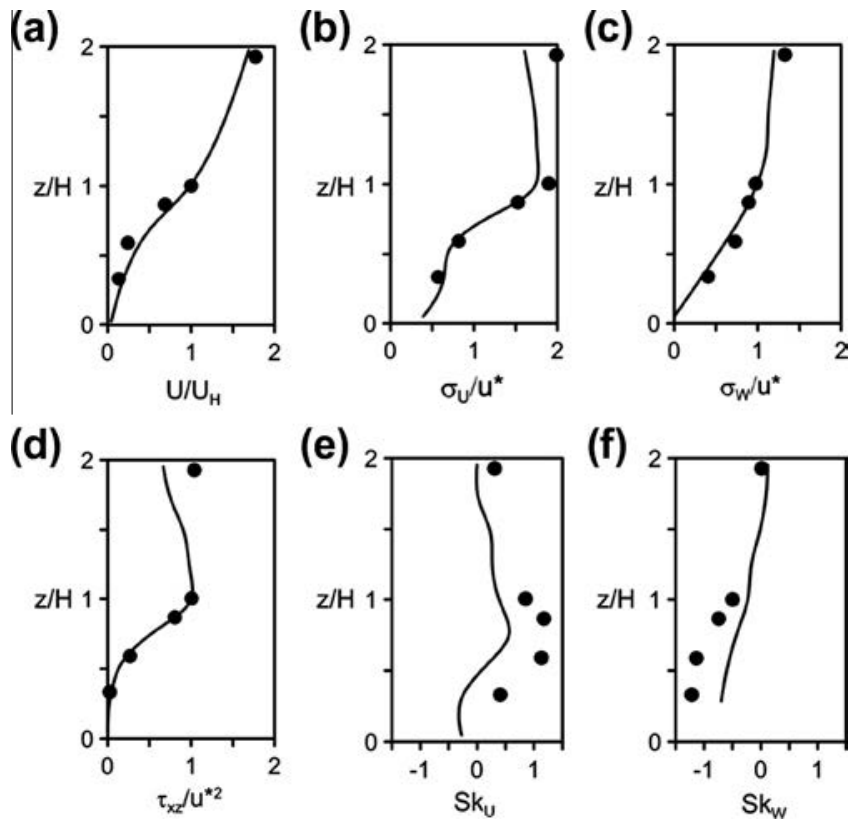

Fig. 2. Vertical profiles of statistical moments compared to experimental data [23] (the dots): (a) the average streamwise velocity, (b) the standard derivation of the streamwise velocity, (c) the standard derivation of the vertical velocity, (d) the Reynolds stress tensor, (e) the skewness of the streamwise velocity, and (f) the skewness of the vertical velocity. 


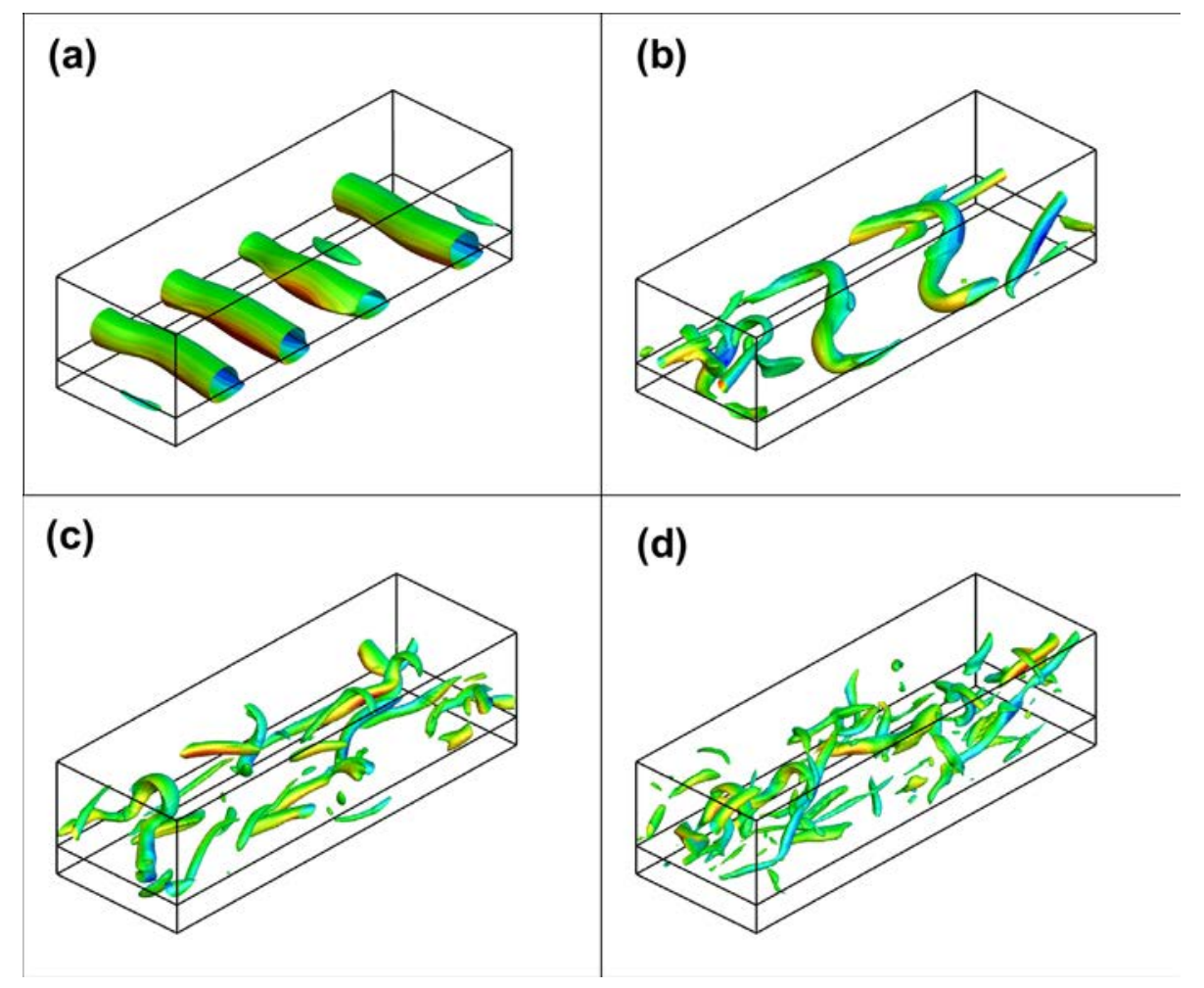

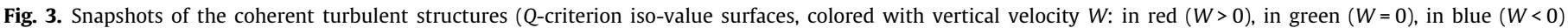

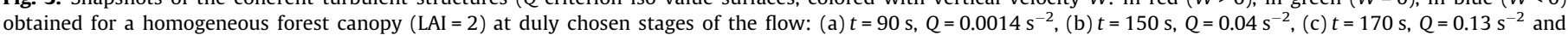
(d) $t=200 \mathrm{~s}, Q=0.15 \mathrm{~s}^{-2}$. (For interpretation of the references to color in this figure legend, the reader is referred to the web version of this article.)

a mixing layer flow. Usually for a flow above surface roughness layer, the standard deviations of the wind components are normalized by the friction velocity $u^{*}$ given by $u^{* 2}=\tau_{X Z}(H)$. In Fig. $2 \mathrm{~d}$, the momentum flux $\tau_{X Z}$ normalized by $u *$ reaches a standard constant stress zone above the canopy and decays rapidly inside the canopy due to the aerodynamic drag absorption of the foliage. In the present study, the profiles of the normalized streamwise and of the vertical velocity RMS increase with height inside the canopy and reach asymptotic values over the canopy. These values $\left(\sigma_{U} / u^{*} \sim 2\right.$ and $\sigma_{W} / u^{*} \sim 1$ ) are typical for turbulence over canopies [2]. The third order statistical moments (skewness) given by $S k_{U}=\left\langle u^{\prime 3}\right\rangle / \sigma_{U}^{3}$ and $S k_{W}=\left\langle w^{\prime 3}\right\rangle / \sigma_{W}^{3}$ constitute a measure for the symmetry of the probability density function associated with the velocity field. A positive skewness indicates an intermittent effect towards larger values, while a negative one reflects an intermittent effect towards smaller values. As shown in Fig. 2e and f, inside the canopy, the skewness is positive for the streamwise velocity component and negative for vertical velocity component. This indicates that turbulence above a forest cover is dominated by intermittent downward moving gusts. At the surface layer far enough from the canopy, the skewness of both the vertical and the streamwise velocities are close to the reference zero value, which corresponds to a Gaussian distribution. We can conclude that the present numerical results are in a relative good agreement with experimental data [23] as shown in Fig. 2. Nevertheless, a noticeable discrepancy was observed for the skewness in Fig. 2e and f. Similar behaviors of skewness were observed in other numerical simulations [16], for which the magnitudes of the skewness obtained from LES were about $50 \%$
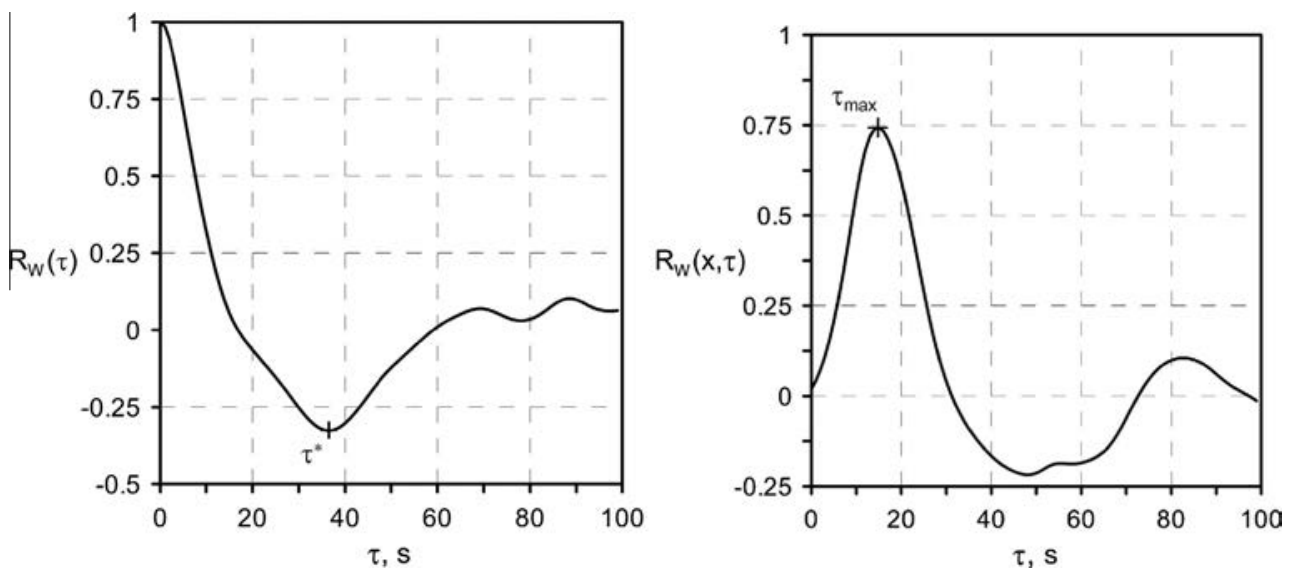

Fig. 4. Autocorrelation (left) and two-point correlation (right) functions of the vertical velocity. 


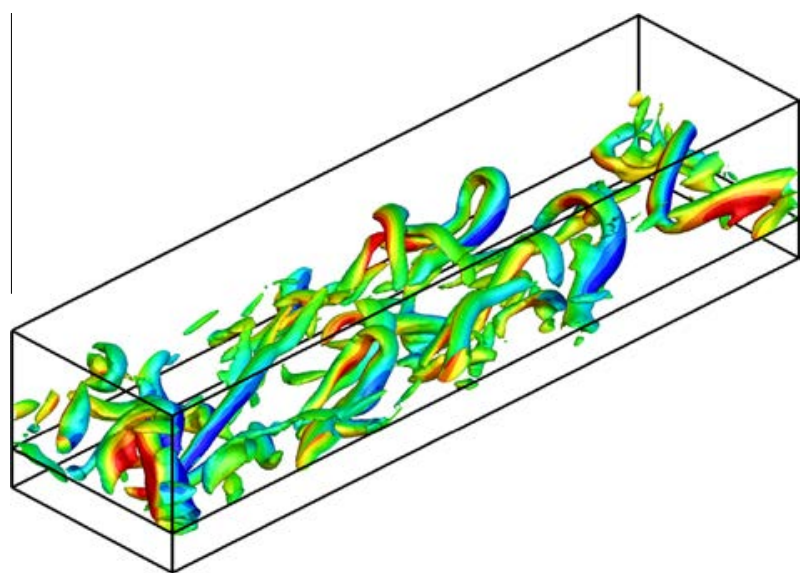

Fig. 5. Coherent large scale structures obtained for a more dense canopy (LAI $=6$ ) shown by $Q$-criterion iso-value surfaces colored by vertical velocity $W\left(Q=0.1 \mathrm{~s}^{-2}\right)$ (red: $W>0$, green: $W=0$, blue: $W<0$ ). (For interpretation of the references to color in this figure legend, the reader is referred to the web version of this article.)

lower than those of the experiments. The authors explained such behavior by the limitations introduced by the grid resolution and the artificial vertical confinement resulting from a non-fully appropriate boundary condition imposed at the upper limit of the computational domain.

An extension of the computational domain in the vertical direction would have probably reduced this artifact.
Iso-value surfaces of the $Q$-criterion were used to visualize large scale coherent structures. These surfaces have been colored with the vertical velocity $W$ (red: $W>0$, green: $W=0$, blue: $W<0$ ). This scalar variable, defined from the rotation-rate tensor and strainrate one, is given by [25]:

$Q=\frac{1}{8}\left[\left(\frac{\partial\left\langle u_{i}\right\rangle}{\partial x_{j}}-\frac{\partial\left\langle u_{j}\right\rangle}{\partial x_{i}}\right)^{2}-\left(\frac{\partial\left\langle u_{i}\right\rangle}{\partial x_{j}}+\frac{\partial\left\langle u_{j}\right\rangle}{\partial x_{i}}\right)^{2}\right]$

The flow evolution leading to fully developed turbulence can be characterized by various stages, outlined in [2], and observed in the present simulations:

- First, as predicted by the inviscid linear stability theory, the existence of an inflection point in the mean streamwise velocity profile $\left\langle u_{1}(z)\right\rangle=U(z)$ promotes the development of a $\mathrm{KH}$ instability. The growth rate and the wavelength of the coherent structures (characterizing this shear instability), are proportional to the magnitude of the shear length $L_{S}$. This quantity is defined as the ratio between the average streamwise velocity with the vertical shear rate evaluated at the top of the canopy as in [3]:

$$
L_{S}=\frac{U(H)}{\left.(d U / d z)\right|_{z=H}}
$$

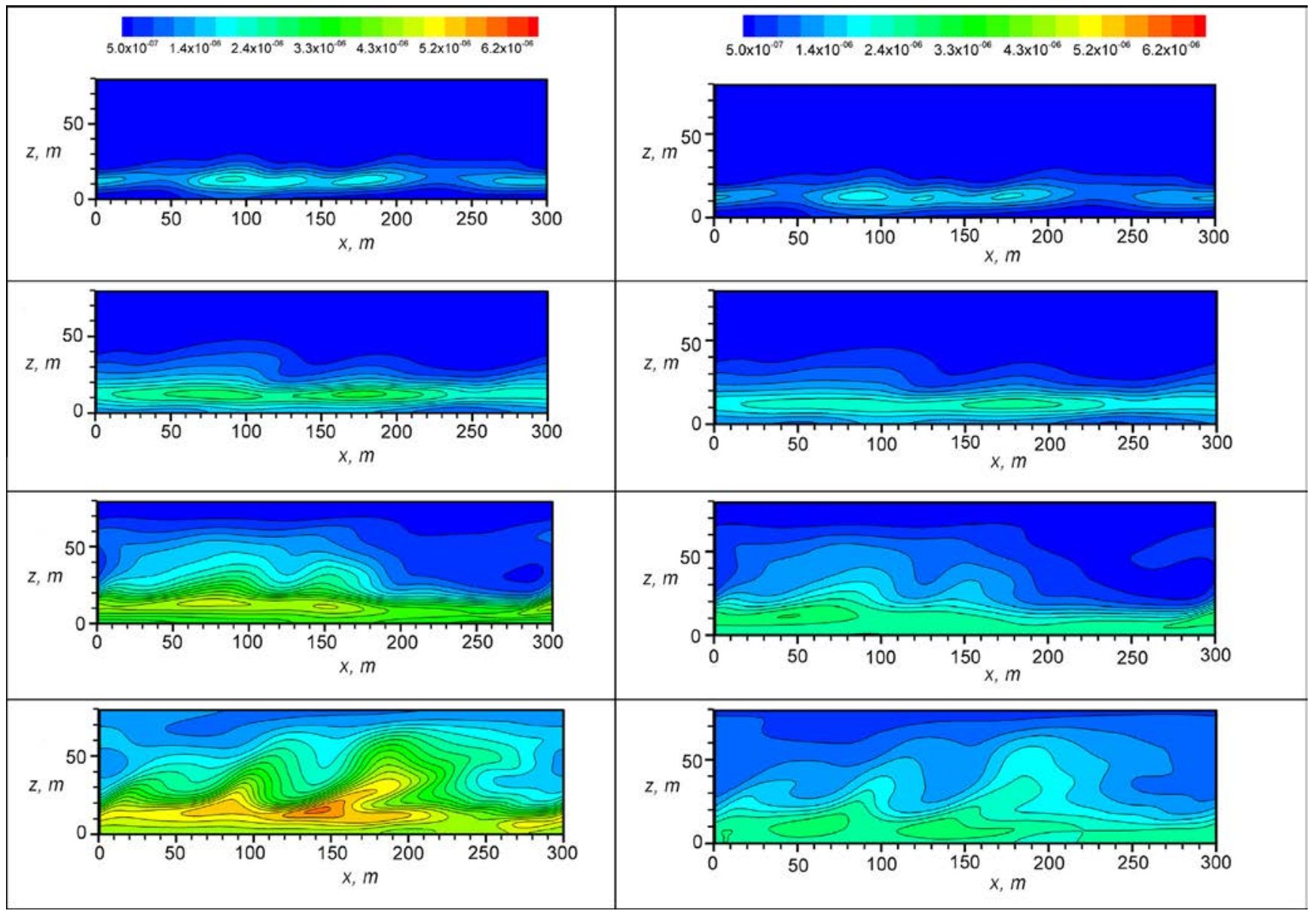

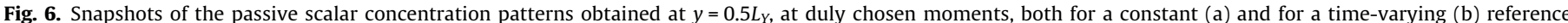

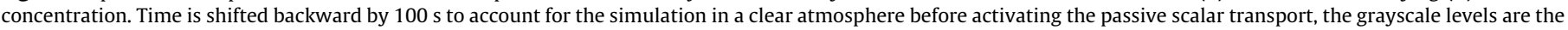
same for both cases (a) and (b). 
- The second stage is the vorticity clumping of the $\mathrm{KH}$ waves into transverse vortices connected by braid regions of highly strained fluid (Fig. 3a). These rolls are transported by convection through the domain at the canopy top level, with a velocity more or less equal to the average ambient wind speed.

- Third, due to a secondary instability, the transverse rolls are transformed from two dimensional patterns into three dimensional ones. The vortex pairing process was also observed in the current computations, but not shown by Fig. 3. At the same time, the transverse rolls are kinked, forming arc-shaped vortices (Fig. 3b).

- The present simulation captures also typical wall turbulence structure, the so-called horseshoe vortex (Fig. 3c). It consists of two counter-rotating quasi-streamwise vortices (legs) connected by an arch vortex (horseshoe). These particular coherent structures play an important role in boundary layer flow. They result from stretching, induced by the ambient deformation field, and from the weak vorticity that exists initially in the stagnation region between $\mathrm{KH}$ billows.

- Finally, the fully developed flow (Fig. 3d) contains all the typical features of canopy turbulence. The fully-developed turbulent canopy flow is characterized by the presence of random structures which are oriented along the streamwise direction. Since the review carried out by Finnigan [2], it is well known that the presence of such longitudinal vortices promotes the succession of weak intensity ejections followed by strong sweeps. This particular flow behavior indicates also the existence of pairs of counter-rotating vortices. Fig. 3c shows the shape of these elongated vortices along the streamwise direction.

Large coherent structures, characterizing the canopy turbulence, can be described using a typical length scale $\Lambda_{X}$ along the streamwise direction. Raupach et al. [3] compared the ratio $\Lambda_{X} / L_{S}$ over a wide range of canopy types and obtained the following relationship:

$\Lambda_{X}=(8.1 \pm 0.3) L_{S}$

For fully developed mixing layer flows the ratio between these two variables is ranged as following: $7<\Lambda_{X} / L_{S}<10$, while the linear stability theory predicted a value $\Lambda_{X} / L_{S} \sim 14$. This result is a new confirmation that the process controlling the generation of large coherent structures in the roughness sub-layer, is very similar to that observed for a plane mixing layer. Using an exponential approximation of the streamwise velocity profile (Fig. 2a), for values calculated inside the canopy $(0<z<H)$, we found that the shear length scale $L_{S}$ was equal to $8.22 \mathrm{~m}\left(L_{S}=0.411 \times H\right)$. This value is close to previous LES results $\left(L_{S}=0.3 \times H\right)$ reported in [26] and also compared to experimental data. As suggested in [3], assuming a Taylor's 'frozen turbulence' assumption, a mean streamwise separation of coherent structures $\Lambda_{X}$ can be approximated from the convective velocity $U_{C}$ (i.e. the displacement velocity of large scale coherent structures) and the time period $\tau$ characterizing large scale structures localized at $z=H$. We introduce the autocorrelation function $R_{W}(\tau)$ and the two-points correlation function $R_{W}(x, \tau)$ of the vertical velocity component (see Fig. 4) (the upper bar indicating a time averaging). The local negative minimum of the autocorrelation function in Fig. 4 (left) indicates the typical time interval $\tau$ that characterized the typical 'period' of coherent structure in fully developed turbulence. Assuming a Taylor's 'frozen turbulence' assumption, the estimation of the streamwise separation distance of coherent structures, $\Lambda_{X}$, can be done by evaluating the convective velocity $U_{C}$. This characteristics velocity can be evaluated from the analysis of the twopoints correlation function $R_{w}(x, \tau)$. To compute this function we need information about two temporal signals of the vertical velocity $w(t)$ and $w(x, t)$ that are derived from two points at a distance $x$ along the streamwise direction. The local maximum of $R_{w}(x, \tau)$, observed in Fig. 4 (right), corresponds to the period of time $\tau_{\max }$. This time describes the interval that is necessary to transport the vortex over a distance $x$. In using all these elements, the streamwise length scale $\Lambda_{X}$ characterizing the average distance separating two coherent structures along the streamwise direction, was evaluated as follows:

$$
\begin{gathered}
R_{W}(\tau)=\frac{\overline{w(t) w(t+\tau)}}{\overline{w^{2}(t)}} \quad R_{w}(x, \tau)=\frac{\overline{\overline{w(t) w(x, t+\tau)}}}{\sqrt{\overline{w^{2}(t)}} \sqrt{\overline{w^{2}(x, t+\tau)}}} \\
\Lambda_{X}=U_{C} \tau^{*}=\frac{x \tau^{*}}{\tau_{\max }}=\frac{40 \times 36.5}{14.8}=98.6 \mathrm{~m}
\end{gathered}
$$

Using this method, we found a ratio $\Lambda_{X} / L_{S}=12$, which exceeds by about $50 \%$ the experimental value $\left(\Lambda_{X} / L_{S}\right)_{\exp }=8.1$ reported in [3]. As indicated in [16], this discrepancy results from the different methods used to estimate the convective velocity $U_{C}$. In the present paper, we used a two-points correlation approach because we considered it to be more accurate than the method consisting in evaluating the integral time-scale from the frequency spectra.

By increasing the drag effects in introducing a more dense canopy (LAI = 6), we can show more clearly (Fig. 5) the structure of the flow formed by two layers of head-down (near the trees top and inside the canopy) and head-up hairpin vortices (above the top of the canopy), as recently suggested by Finnigan et al. [16]. As indicated in this same paper, the superposition of these two types of hairpin vortices, allowed to understand how, inside and near the top of the canopy, the turbulence was dominated by Q4-sweep events (promoted by head-down hairpin vortices), and far enough the same turbulence was dominated by Q2-ejection events (promoted by head-up horseshoe vortices).
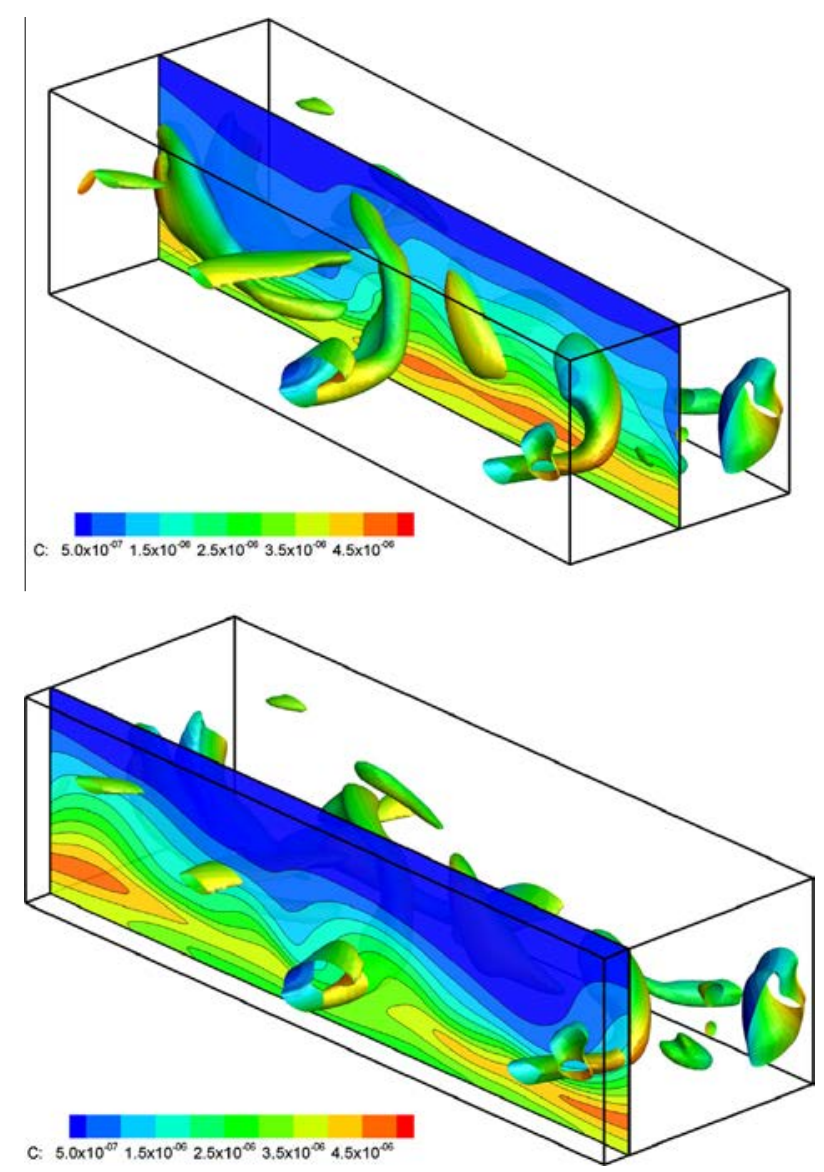

Fig. 7. Large scale coherent structures obtained at $t=200 \mathrm{~s}$, shown by $Q$-criterion iso-value surfaces and colored by the passive scalar concentration field. 


\section{Passive scalar dispersion}

The following results were obtained using the same configuration (shown in Fig. 1) and under the same conditions of simulation, as in the previous section, but in activating Eqs. (5) and (6), and thus in solving the transport equation for the passive scalar. The simulation started with a clear atmosphere, i.e. the initial distribution of the passive scalar concentration was $C=0$, and the initial reference concentration at the leaf surface was proportional to the LAD: $C_{\text {ref }}=a_{L} \times 10^{-4}$. It is important to note that Eqs. (5) and (6) were activated only after about $100 \mathrm{~s}$ of simulation in a clear atmosphere, which was enough time for the flow to reach a fully developed turbulent state. As mentioned earlier, two cases have been considered: (1) the reference concentration $C_{\text {ref }}$ was constant and (2) the reference concentration varied with time according to Eq. (6).

Fig. 6 shows snapshots of the scalar concentration patterns obtained in the vertical median plane, i.e. $y=0.5 L_{Y}$, at duly chosen moments of simulation, both for a constant and for a time-varying reference concentration. At the beginning of the transport process, maximum concentration values in the air were observed near $z=0.5 \mathrm{H}$ where the reference concentration inside canopy was the highest and the concentration magnitudes were similar for both considered cases. Then, lower concentration magnitudes were observed in case (2) - where $C_{\text {ref }}$ varied with time - in comparison to case (1). It is worth noting here that due to the periodic boundary conditions in the streamwise direction, the passive scalar concentration in the domain did not dissipate, which would not be the case if Neumann conditions were applied for the concentration field at the domain inlet and outlet. Fig. 6 shows also the concentration patterns that were responsible for the transport of passive scalar from the canopy into the upper atmosphere. These fingershape patterns, inclined by $40^{\circ}$ with respect to the streamwise direction, are due to the well-known streaks encountered in boundary layer flows [14].

The coupling between the dynamics of coherent structures (studied in part 4) with the passive scalar transport was clearly highlighted in Fig. 7, showing at the same time Q-criterion iso-value surfaces and passive scalar concentration field. This figure shows without any ambiguity that it was the large scale structures, containing the major part of the kinetic energy, which contributed the most to the mass transfer between the canopy and the atmosphere, as well as to the mass transport process through the atmospheric boundary layer.

Fig. 8 shows the time evolution of the passive scalar concentration in the atmosphere and that of the reference concentration along the vertical line $\left(x=L_{X} / 2\right.$ and $\left.y=L_{Y} / 2\right)$ at positions $(z=0.3 H$, $0.8 \mathrm{H}, 1.3 \mathrm{H})$. As the position height $\mathrm{z}$ increases, the intensity of turbulence increases and the oscillation amplitudes of the passive scalar concentration increase as well. According to Eq. (6), Fig. 8b and (a)

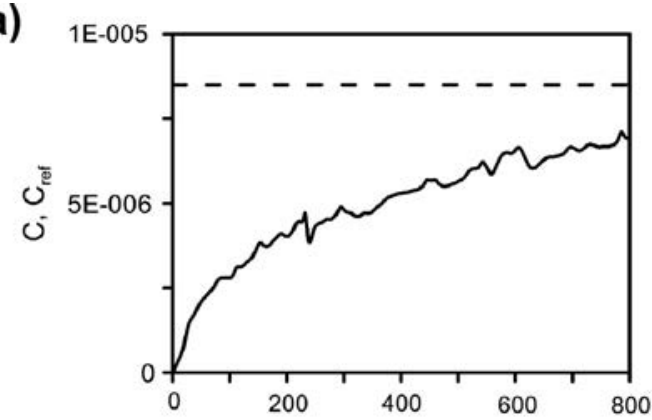

(c)

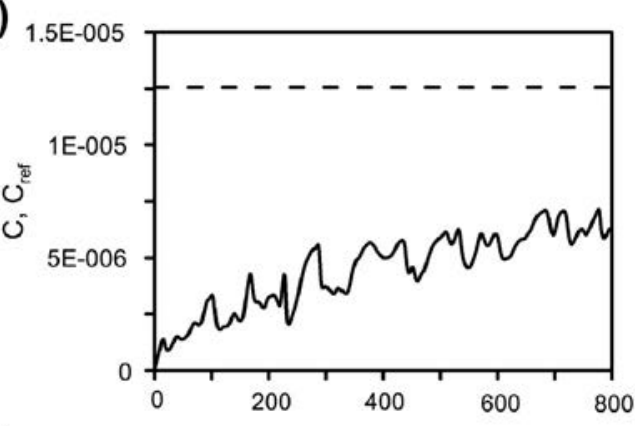

(e)

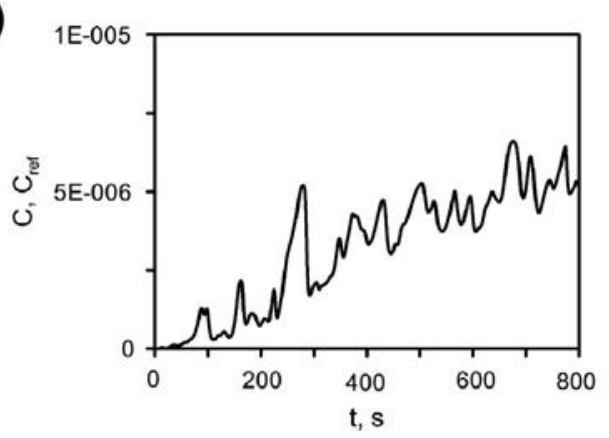

(b)

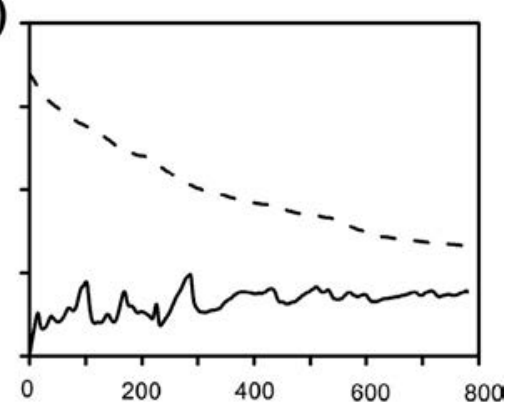

(d)

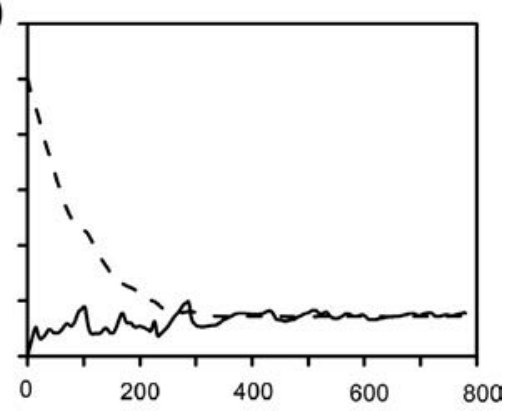

(f)

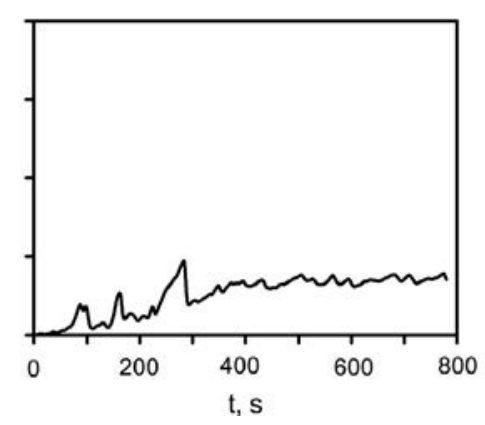

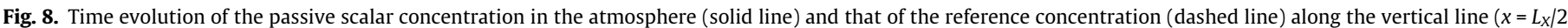

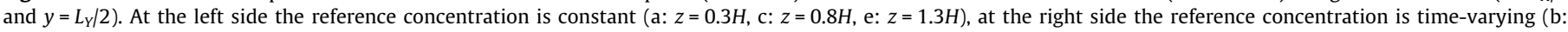

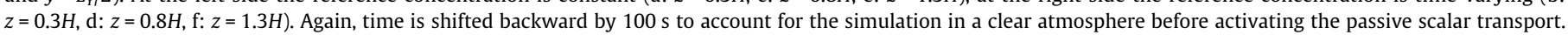


(a)

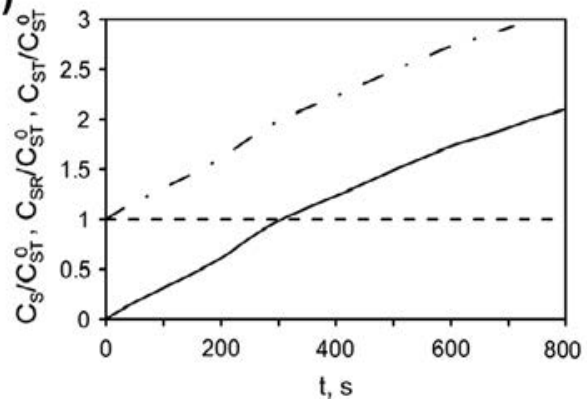

(b)

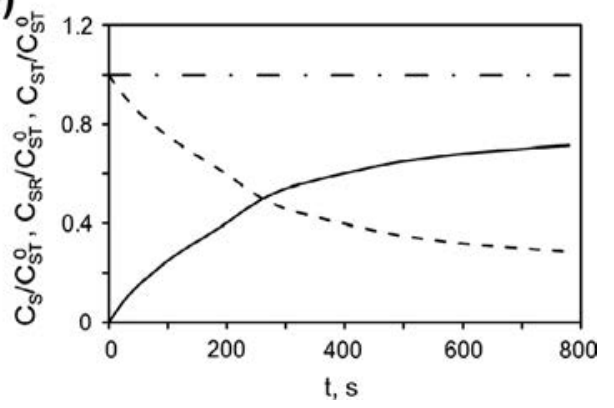

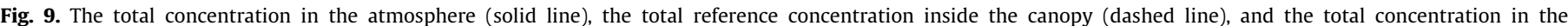

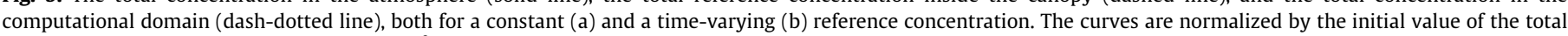
concentration in the computational domain $C_{S T}^{0}$.

d show a tendency of the passive scalar concentration in the atmosphere and the reference one to become equal. By comparing Fig. 8e and f, we notice again as expected, that the passive scalar concentration in the case of a time-varying reference concentration (case 2) was several times smaller than in case 1 (constant reference concentration).

The total concentration of the passive scalar in the atmosphere $C_{S}$, the total reference concentration inside the canopy $C_{S R}$, and the total concentration in the computational domain $C_{S T}$ are defined by Eqs. (11)-(13) respectively, where $D$ represents the computational domain. Note that $C_{\text {ref }}$ is zero outside the canopy.

$C_{S}=\int_{D} C d x d y d z$

$C_{S R}=\int_{D} C_{\text {ref }} d x d y d z$

$C_{S T}=\int_{D}\left(C+C_{\mathrm{ref}}\right) d x d y d z$

As shown in Fig. 9, in the case where the reference concentration was maintained to be constant, the total concentration in the atmosphere increased indefinitely, as a consequence of the infinite source of passive scalar coming from the surface of the foliage of the canopy. However in the case (2), the total concentration in the atmosphere increased also as a function of time but the total reference concentration inside canopy decreases by the same amount (according to Eq. (6)), in such a way that the sum of this two contributions remained constant.

In case (1) where the reference concentration was constant, the curve of $C_{S}$ was close to the straight line. The fact that $C_{S}$ exceeded $C_{S R}$ and keep growing quasi-linearly with time means that the total concentration in the atmosphere up to $z=H$ remained smaller than $C_{S R}$. So the time derivative of the total concentration in the atmosphere was approximately constant in this case (according to Eq (6)) and did not depend on $C_{S}$, i.e. $d C_{S} / d t \approx c_{C} \tilde{a}_{L} \tilde{\rho} \tilde{u} C_{\mathrm{SR}}$, where $\tilde{a}_{L}$, $\tilde{\rho}$, and $\tilde{u}$ were respectively the average values of the LAD, of density, and of the effective streamwise velocity inside the canopy.

In case (2) where the reference concentration varied in time, the linear part of the time evolution of $C_{S}$ was much shorter then in case (1); the limitation factor was more important in this case as $C_{S R}$ decreased. The following exponential approximations for the total concentrations, in good agreement with numerical results, could be proposed in case (2):

$C_{S} / C_{S T}^{0}=A\left(1-e^{-t / \tau}\right)$,

$C_{S R} / C_{S T}^{0}=(1-A)+A e^{-t / \tau}$,

where $A$ is the normalized steady-state total concentration in the atmosphere $(A \approx 0.73)$ and $\tau$ is the typical relaxation time
( $\tau \approx 220 \mathrm{~s}$ ). According to Eq. (6), the typical relaxation time could be estimated as $\tau=1 / c_{C} \tilde{a}_{L} \tilde{\rho} \tilde{u}$. Given the vertical distribution of $a_{L}$ :

$\tilde{a}_{L}=\frac{1}{H} \int_{0}^{H} a_{L} d z=\frac{\text { LAI }}{H}=0.1$

From the estimated value of the relaxation time $\tau$, we deduced that the effective streamwise velocity $\tilde{u} \approx 1.1 \mathrm{~m} / \mathrm{s}$, which corresponds to the average velocity at $\mathrm{z}=0.7 \mathrm{H}$. These values of $\tilde{a}_{L}$ and $\tilde{u}$ exhibited also a very good agreement for the slope of $C_{S}(t)$ obtained in case (1) where the reference concentration was kept constant.

\section{Conclusion}

The problem of interaction between an ABL flow and a forest canopy was studied numerically using large eddy simulations (LESs). The numerical results confirmed that this flow presented many similarities with a mixing layer flow. Snapshots of the coherent structures have shown in using iso-value surfaces of the $Q$-criterion, allowed to highlight the scenario and the physical mechanisms contributing to the final organization of this turbulent flow. In the present study, KH rolls were formed at the canopy top due to the shear nature of the flow. The kinking and pairing of $\mathrm{KH}$ rolls were similar to those occurring in a mixing layer flow. Since the flow has demonstrated also similarities with a turbulent boundary layer, horseshoe vortex formation could also be observed above the canopy. The shear induced by the action of the vegetation upon the flow resulted in large-scale coherent structures mainly located at the canopy-free flow interface. The vertical profiles of the average flow velocity, of the velocity standard derivations, and of the skewness were all in good agreement with previously reported results obtained in a fully developed turbulent flow. A good agreement was also observed between the results obtained using LES and experimental data concerning turbulence characteristics. The transport of a passive scalar was not only seen in this paper as a signature of the passage of coherent turbulence structures as in [12-14], resulting in the previously reported ramp patterns that were observed again in the present study. The model presented in [14] has been modified in order to take into account a time evolution of the concentration field at the surface of foliage, hence modeling a more realistic pollutant exchange between a contaminated canopy and an initially clear atmosphere. Despite the fact that this new model has little influence on the concentration ramp-patterns, it exhibits a significant effect on the concentration magnitude that was dramatically decreased, as well as on the dynamics of the total passive scalar concentration in the atmosphere: growing linearly with time in case (1) where the reference concentration was kept constant, and growing exponentially with time and reaching about $73 \%$ of the total initial concentration in- 
side the canopy in case (2) where the reference concentration was time-varying according to a kinetic equation.

\section{Acknowledgements}

The authors thank the financial support of the European research project FIREPARADOX in the context of which the investigations presented in this paper have been conducted. The authors also thank the anonymous reviewers for their constructive suggestions, which allowed to substantially improving the present paper.

\section{References}

[1] Kaimal JC, Finnigan JJ. Atmospheric boundary layer flows. Oxford, UK: Oxford University Press; 1994. p. 289.

[2] Finnigan J. Turbulence in plant canopies. Annu Rev Fluid Mech 2000;32:519-71.

[3] Raupach MR, Finnigan JJ, Brunet Y. Coherent eddies and turbulence in vegetation canopies: the mixing-layer analogy. Bound - Layer Meteorol 1996;78:351-82.

[4] Massman WJ. A comparative study of some mathematical models of the mean wind structure and aerodynamic drag of plant canopies. Bound - Layer Meteorol 1987; 40:179-97.

[5] Ghisalberti M, Nepf HM. Mixing layers and coherent structures in vegetated aquatic flows. J Geophys Res 2002;107(2):1-11.

[6] Denmead OT, Bradley EF. On scalar transport in plant canopies. Irrig Sci 1987;8:131-49.

[7] Gao W, Shaw RH, Paw UKT. Observation of organized structure in turbulent flow within and above a forest canopy. Bound - Layer Meteorol 1989;47:349-77.

[8] Deardorff JW. Numerical investigation of neutral and unstable planetary boundary layers. J Atmos Sci 1972;29(1):91-115.

[9] Moeng CH. A large eddy simulation model for the study of planetary boundarylayer turbulence. J Atmos Sci 1984;41:2052-62.

[10] Shaw RH, Schumann U. Large-eddy simulation of turbulent flow above and within a forest. Bound - Layer Meteorol 1992;61:47-64.

[11] Kanda M, Hino M. Organized structures in developing turbulent flow within and above a plant canopy, using a large-eddy simulation. Bound - Layer Meteorol 1994;68:237-57.
12] Finnigan JJ, Shaw RH. A wind-tunnel study of airflow in waving wheat: an EOF analysis of the structure of the large-eddy motion. Bound - Layer Meteorol 2000;96:211-55.

[13] Fitzmaurice L, Shaw RH, Paw UKT, Patton EG. Three-dimensional scalar microfront systems in a large-eddy simulation of vegetation canopy flow. Bound - Layer Meteorol 2004;112:107-27.

[14] Watanabe T. Large-eddy simulation of coherent turbulence structures associated with scalar ramps over plant canopies. Bound - Layer Meteorol 2004;112:307-41.

[15] Huang M, Cassiani M, Albertson JD. The effects of vegetation density on coherent turbulent structures within the canopy sub-layer: a large-eddy simulation study. Bound - Layer Meteorol 2009;133:253-75.

[16] Finnigan JJ, Shaw RH, Patton EG. Turbulence structure above vegetation canopies. J Fluid Mech 2009;637:387-424.

[17] Su H-B, Shaw RH, Paw UKT, Moeng C-H, Sullivan PP. Turbulent statistics of neutrally stratified flow within and above a sparse forest from large-eddy simulation and field observations. Bound - Layer Meteorol 1998;88: 363-97.

[18] Schumann U. Subgrid scale model for finite difference simulations of turbulent flows in plane channels and annuli. J Comput Phys 1975;18:376-404.

[19] Isaa RI. Solution of the implicitly discretized fluid flow equation by operator splitting. J Comput Phys 1986;63:40-65.

[20] Accary G, Meradji S, Morvan D, Fougere D. Towards a numerical benchmark for 3D mixed-convection low mach number flows in a rectangular channel heated from below. J Fluid Dyn Mater Process 2008;141(1):1-7.

[21] Accary G, Bessonov O, Fougere D, Meradji S, Morvan D. Optimized parallel approach for 3D modelling of forest fire behaviour. Lect Notes Comput Sci 2007;4671:96-102.

[22] Launder BE, Spalding DB. The numerical computation of turbulent flows. Comp Meth Appl Mech Eng 1974;3:269-89.

[23] Shaw RH, Den Hartog G, Neumann HH. Influence of foliar density and thermal stability on profiles of Reynolds stress and turbulence intensity in a deciduous forest. Bound - Layer Meteorol 1988;45:391-409,.

[24] Hu X, Lee X, Stevens RH, Paw UKT, Smith RB. A numerical study of nocturnal wavelike motion in forests. Bound - Layer Meteorol 2002;102:199-223.

[25] Hunt J, Wray A, Mion P. Eddies, streams and convergence zones in turbulent flows. Center of Turbulence Research Rep. CTR-S88; 1988. 193p.

[26] Yue W, Meneveau Ch, Parlange MB, Zhu W, Kang HS, Katz J. Turbulent kinetic energy budgets in a model canopy: comparisons between LES and windtunnel experiments. Environ Fluid Mech 2008;8:73-95. 